\title{
APROXIMACIÓN A LOS CONCEPTOS DE CONOCIMIENTO, CREENCIA E IDEOLOGÍA EN LA ENSEÑANZA DE LA HISTORIA DE VENEZUELA A PARTIR DE LOS APORTES DE TEUN VAN DIJK
}

\section{APPROACH TO THE CONCEPTS OF KNOWLEDGE, BELIEF AND IDEOLOGY IN THE TEACHING OF THE HISTORY OF VENEZUELA FROM THE CONTRIBUTIONS OF TEUN VAN DIJK}

\begin{abstract}
María Elena Del Valle Mejías ${ }^{1}$ (Universidad Metropolitana de Caracas)
\end{abstract}

manedelvalle@gmail.com

\section{RESUMEN}

El presente artículo analiza los conceptos de conocimiento, creencia e ideología en la enseñanza de la Historia de Venezuela desarrollados en los textos escolares. El análisis se hace a partir de los aportes teóricos de Teun van Dijk que en su prolífica obra aborda los mencionados constructos y como estos pueden ser analizados en diferentes discursos, en este caso, en un discurso concreto que es el académico. Se compilan ejemplos concretos en los cuales pueden observarse el específico tratamiento de las categorías de análisis mencionadas.

Palabras Clave: análisis del discurso, creencia, ideología, Historia de Venezuela.

\footnotetext{
${ }^{1}$ María Elena Del Valle Mejías, nacida en Caracas, Venezuela, el 10 de marzo de 1970, graduada de Profesora en la Universidad Pedagógica Libertador en el año 1992, con especialización en Historia Económica y Social de Venezuela en la Universidad Santa María, con Maestría en Historia Económica y Social de Venezuela, tesis con mención publicación y suma cum laude, Doctora en Ciencias de la Educación en el Pedagógico de Caracas, con postdoctorado en Semiótica y Pragmática en la Universidad Complutense de Madrid, cuenta con proyectos financiados por la UPEL y con participación de la Universidad Complutense de Madrid, con proyectos financiados por el FONACIT, investigadora certificada de la Universidad Complutense de Madrid, el grupo internacional CONCILIUM y del CELARG, ponente regular en eventos nacionales e internacionales, adscrita a la línea de investigación, Ciencias del lenguaje y con siete años de investigación en el área de análisis del discurso. Profesora Ordinaria de la Universidad Pedagógica Libertador. Investigadora acreditada del CYTED, la AECID, profesora de la UNIMET.
} 


\section{ABSTRACT}

This article analyzes the concepts of knowledge, belief and ideology in the teaching of Venezuelan History developed in school textbooks. The analysis is based on the theoretical contributions of Teun van Dijk who in his prolific work addresses the aforementioned constructs and how they can be analyzed in different discourses, in this case, in a specific discourse that is academic. Concrete examples are compiled in which the specific treatment of the categories of analysis mentioned can be observed.

Keywords: discourse analysis, belief, ideology, History of Venezuela.

\section{Cómo citar el artículo:}

María Elena Del Valle Mejías. (2013) APROXIMACIÓN A LOS CONCEPTOS DE CONOCIMIENTO, CREENCIA E IDEOLOGÍA EN LA ENSEÑANZA DE LA HISTORIA DE VENEZUELA A PARTIR DE LOS APORTES DE TEUN VAN DIJK. Revista de Ciencias de la Comunicación e Información. (Diciembre 2013). Año XXVII (30), 50-58 ISSN: 0213-070-X. Recuperado de:

\section{Los grandes conocimientos engendran las grandes dudas. Aristóteles.}

A lo largo de toda su formación el joven venezolano estudia y aprende una gran cantidad de conocimientos relativos a la Historia. Dicha asignatura acompaña la educación del joven durante los seis años de la educación primaria y los cinco del bachillerato. En esta ocasión, y a la luz de los aportes de Van Dijk en su obra Ideología (2006), pretendo reflexionar en torno a las implicaciones que desde su perspectiva y en el contexto específico de la enseñanza de la Historia tiene eso que Ilamamos conocimiento. El presente estudio se ubica dentro del área del análisis crítico del discurso como práctica que busca develar la presencia de ideologías en los discursos, en este caso el discurso pedagógico en la enseñanza de la Historia.

Van Dijk (2006) define como conocimiento el producto del pensamiento que se considera verdadero. El autor establece, además, que el conocimiento es una categoría de la creencia, a saber, aquellas creencias que nosotros (como grupo, comunidad, cultura...) consideramos creencias verdaderas, de acuerdo con ciertos fundamentos o criterios. De esta manera, continúa el autor, estas 
creencias son válidas, correctas, certificadas, sostenidas de manera general 0 , al menos, reúnen los estándares de verdad socialmente compartidos (p. 35).

Partiendo de estos principios, en Historia consideraremos verdaderos y creíbles aquellos supuestos que desde las exigencias de cada contexto epistemológico reúnan los atributos requeridos. Van Dijk (2006) agrega que estos atributos son social, cultural e históricamente variables, así como lo es el conocimiento basado en ellos (p. 35), de tal manera que lo que ayer fue verdad, tal vez hoy no lo sea.

De tal forma, y basándonos en las afirmaciones del autor, podríamos aseverar que hay diferentes conocimientos históricos de acuerdo con el contexto y las exigencias del mismo. Ahora bien, cuando un estudiante de bachillerato estudia la historia universal o la historia de su país, de manera indirecta o implícita asume que lo que se recoge en ella es creíble.

Este conjunto de creencias (sistema de creencias) como la define el autor, pueden tener diferentes atributos, los cuales explica a través de un ejemplo:

1. El agua se congela a 0 grados centígrados.

2. Ámsterdam es la capital de Holanda.

3. El mes pasado di una conferencia en Valparaíso.

4. Me gusta el helado.

5. Krzysztof es mi vecino.

6. Había una niña en Nicaragua que sonreía mientras montaba un jaguar (p. 47).

En la enumeración presentada por el autor pueden apreciarse diferentes tipos de creencia, todas ellas basadas en cosas de la vida diaria. Lo que me planteo en este caso es cuestionar qué conocimientos adquiridos a través del estudio de la Historia a través del texto o escuchando al docente, podemos ir comparando con la clasificación de Van Dijk (2006). El autor afirma que las de la lista anterior son creencias no ideológicas. En la 1 y la 2, los hechos descritos son indiscutibles, al menos desde el marco del conocimiento que hoy consideramos verdadero. La capital de un país y los grados a los que se congela el agua. En la 3 se hace referencia a hechos pasados, en la 4 a preferencias personales, en la 5 hechos de la vida y en la 6 hechos ficticios o literarios (p. 48).

Hagamos ahora un ejercicio de creencias no ideológicas en Historia:

1. Colón descubrió América.

2. Venezuela limita al norte con el Mar Caribe.

3. José Gregorio Monagas abolió la esclavitud.

4. Hugo Chávez dio un golpe de estado. 
5. No me gustan los indígenas.

Procedo a calificarlas como no ideológicas porque responden a la misma clasificación hecha por el autor. A saber, la 1 y la 2 responden a hechos indiscutibles, la 3 y la 4 hacen referencia a hechos del pasado y la 5 hace alusión a preferencias personales. Sin embargo, y es aquí donde quisiera detenerme, cuando asumimos conocimientos 0 creencias que consideramos como verdaderas y éstas se circunscriben al ámbito del pasado o la reconstrucción histórica, debemos manejar variables distintas.

En la primera afirmación: Colón descubrió América, se cumple lo que Van Dijk (2006) denomina como creencia evaluativa u opinión. La misma es definida como: “(...) decirle a un grupo qué es bueno y qué es malo, correcto e incorrecto" (p. 53). Las creencias, según Van Dijk (2006), validan opiniones personales, en este caso en la Historia de Venezuela han existido diversos abordajes de este hecho "indiscutible". En la segunda mitad del siglo $X X$ se denominaba a este acontecimiento como "el descubrimiento de América".

Si analizamos este calificativo, muy parecido al de la lista de creencias, hay una subestimación implícita al considerar que se descubre un continente. El verbo descubrir implica que hay algo que se desconoce y por lo tanto no existe. América no existe hasta el momento en el cual Colón y el imperio español llegan a ella. No es necesario hacer apología de las altas culturas indígenas y de los notables y hoy bien reconocidos aportes que han legado a la humanidad y tampoco de eso se trata, lo que pretendo es evidenciar cómo en una creencia sin aparente carga ideológica pueden imbricarse afirmaciones que hacen ideología.

Van Dijk (2006) afirma que las ideologías no son metafísicas, por el contrario están firmemente localizadas, no por encima ni entre las personas, sino que son parte de sus miembros (p. 71). Esta creencia no ideológica, a mediados de los ochenta cambió su estructura semántica y denominó a este hecho indiscutible con otros calificativos. La llegada de Colón a América comenzó a definirse como "el encuentro de dos mundos". Ahora se le concedía a los pueblos de América partida de nacimiento antes de la llegada del imperio, ya no se trataba de un descubrimiento sino del encuentro entre dos culturas, dos mundos concretos y diferentes. Ahora, en pleno siglo XXI, y bajo los matices de la Revolución Bolivariana, ese mismo hecho indiscutible cambia de calificativo.

Las reflexiones de los historiadores desde y para una ideología, denominan ahora a este hecho "el día de la resistencia indígena". Como puede verse, tan sólo en la escogencia de las palabras que describen un hecho indiscutible la pertenencia o el compromiso con una ideología determinada puede ponerse en evidencia. Se trata, como 
afirma Van Dijk (2006), de opiniones importantes para la interacción, coordinación y reproducción del grupo, opiniones que se basan en valores y principios variables de grupo a grupo (p. 73). De esta manera una misma afirmación, como envoltura inmaterial aparentemente ingenua, tiñe de posturas ideológicas su descripción.

Se puede usar y abusar del conocimiento, afirma Van Dijk (2006); se le puede aplicar para controlar a la gente (p. 73). Cabría entonces preguntarse si al hacer afirmaciones 0 al repetir creencias aparentemente no ideológicas, se legitiman o deslegitiman visiones de la realidad, que el alumno aprende, repite y memoriza a lo largo de su formación.

Veamos ahora la segunda creencia: Venezuela limita al norte con el Mar Caribe. Van Dijk (2006) denomina a este tipo de creencia como "fáctica" y establece una clasificación de la misma creencia en verdadera o falsa. El autor describe los atributos de las creencias fácticas usando el ejemplo de la afirmación número 2: Ámsterdam es la capital de Holanda. Las creencias fácticas no implican evaluación, de tal manera que son verdaderas o falsas si se ajustan a los criterios de verdad objetivos socialmente establecidos.

Sin embargo el mismo Van Dijk concede que esa misma afirmación pueda ser utilizada evaluativamente, como en la acusación: "Ámsterdam es la capital de las drogas" (p. 55). Podemos concluir, junto con el autor, que en ciertas áreas del saber es realmente muy difícil hacer explícita la distinción entre conocimiento y opinión, entre conocimiento y evaluación. En muchos casos tendrá entonces que ver con lo avanzado del conocimiento en el área, teniendo entonces que aceptar que el conocimiento y lo que asumimos como verdadero cambia.

Revisemos ahora la afirmación número 3: José Gregorio Monagas abolió la esclavitud. En el libro Historia contemporánea de Venezuela de Antonio Gómez (2007) se hace la siguiente reseña del gobierno del citado personaje:

"A pesar de lo incoloro de esta administración, durante este ejercicio se llevó a cabo una determinación del poder ejecutivo que por sí sola vale para llevar a la Historia, con brillo, a José Gregorio Monagas: Ia abolición total y absoluta de la esclavitud" (p. 50).

Van Dijk (2006) afirma que las creencias, fácticas o no, son subjetivas, y por lo tanto pueden ser erróneas, infundadas o desviadas (p. 35), y estas creencias a su vez pueden constituirse en conocimiento cuando se compilan en un libro de texto que el estudiante en el mejor de los casos aprende y memoriza. Revisemos la afirmación del autor en el texto anterior: José Gregorio Monagas es Ilevado a la Historia con brillo gracias a la abolición total y absoluta de la esclavitud. 
La esclavitud en ese contexto histórico es abolida por razones eminentemente económicas:

- La prohibición de importación de esclavos de África.

- La ley de manutención que golpeaba a los dueños de esclavos al obligarlos a mantener a un contingente en diversas necesidades: hogar, vestido, alimento, etc.

- Debido al desgaste físico al que los esclavos eran sometidos, un negro a la edad de 37 años ya era inservible, pero no por eso dejaba de ser obligación del amo mantenerlo.

Éstas, entre otras razones de complejo origen, son las llevaron a la administración de José Gregorio Monagas a la abolición, al menos en el cuerpo constitucional, de la esclavitud, ya que tenemos conocimiento de que las condiciones laborales bajo las cuales el liberto quedó eran aun peores a las de la esclavitud en sí misma. Sin embargo, estas afirmaciones se constituyen en lo que Van Dijk denomina "ladrillos de la mente" (p. 35).

Si el estudiante no cuenta (lo cual es lo más común) con un docente que lo lleve a analizar desde un punto de vista más estructural que coyuntural este hecho, puede dicha afirmación hacer "edificio" en la mente del joven, quien también embalsamará en su concepción de la historia de Venezuela a José Gregorio Monagas como un bienhechor. Nos encontramos, entonces con un discurso, el histórico, en el cual cada palabra, cada adjetivo, cada epíteto puede responder y de hecho responde a ideologías concretas.

Otro elemento a tomar en consideración es lo que Van Dijk (2006) denomina evaluaciones, definidas como lo que nosotros pensamos que es falso o verdadero, agradable o desagradable, permitido o prohibido, aceptable o inaceptable, las cuales son producto de juicios basados en normas o valores. Lo que planteo es que en muchos de los conocimientos históricos que el estudiante aprende hay mucho de creencia, mucho de evaluación, mucho de opinión.

Analicemos ahora la siguiente afirmación o creencia no ideológica: Hugo Chávez dio un golpe de estado. Para analizar esta afirmación es primero necesario definir lo que el autor denomina como una clase de objeto mental que puede ser creencia o no, de acuerdo con la posición teórica que uno sostenga, a saber, el sentimiento o la emoción (p. 37).

Van Dijk usa para esta categoría un ejemplo claro: el genocidio de Bosnia. Sentir enojo o preocupación por el genocidio de Bosnia implica o presupone la creencia de que hay un genocidio en Bosnia, y normalmente también que el genocidio es malo ( $p .37$ ). 
Cuando buscamos en tres libros de texto cómo se describe este hecho histórico encontramos:

Primero, en el texto de Napoleón Franceschi y Freddy Domínguez (2005) se dice como parte de una breve reseña del segundo gobierno de Carlos Andrés Pérez lo siguiente:

"En su segunda presidencia, Carlos Andrés Pérez enfrenta una rebelión popular el 27 y 28 de febrero de 1989 y dos intentonas militares el 4 de febrero y el 27 de noviembre de 1992" (p. 383).

El segundo texto es el de Antonio Gómez (2007) y el hecho es descrito de la siguiente manera:

"La impopularidad de las medidas económicas y su manipulación por parte de grupos interesados en desestabilizar el régimen, trajo como resultado el estallido social que se inició en la ciudad de Guarenas, el 27 de febrero de 1989, y continuó en Caracas (...) dando como saldo un número indeterminado de muertos, violación de derechos humanos y grandes pérdidas materiales (...). El 4 de febrero de 1992 ocurrió el alzamiento fallido encabezado por el teniente coronel Hugo Rafael Chávez Frías, acompañado de Arias Cárdenas, Joel Acosta Chirinos, Jesús Urdaneta y un buen número de efectivos militares" (p. 215).

El tercer texto es el de Alberto Arias Amaro (2000) y el hecho se describe como sigue:

"Cada día se alejaban más las perspectivas de participación democrática del pueblo en el ejercicio del gobierno y crecía la influencia de los 'cogollos partidistas' y de altos sectores de la economía en la orientación política. La agudización de la crisis económica y política y el descontento de la población por las medidas impopulares del gobierno, crearon las condiciones para que surgiera el peligro de un golpe de estado. La conspiración se fraguó en las Fuerzas Armadas y estuvo encabezada por el teniente coronel Hugo Chávez Frías, quien, acompañado por un numeroso grupo de oficiales medios, intentó un golpe de estado el 4 de febrero de 1992" (p. 230).

Ahora analicemos cada uno de los textos en función de lo que podríamos denominar "un hecho indiscutible" que es: Chávez dio un golpe de estado. El evento tiene una fecha, unos personajes, un espacio físico donde se lleva a cabo pero no es presentado de la misma forma. En el caso de Franceschi y Domínguez (2005) el hecho es registrado sin hacer alusión a quién da la intentona golpista, sólo se afirma que Carlos Andrés Pérez enfrenta dos intentonas.

Mientras otros conocimientos son descritos prolijamente, éste es presentado con menos de los datos básicos, ya que ni siquiera el nombre del teniente coronel Hugo Chávez Frías es reseñado. ¿A qué 
se deberá esto? ¿Se tratará de que, como afirma Van Dijk (2006), las ideologías encarnan sentimientos a favor $o$ en contra y esos sentimientos se ven inevitablemente reflejados en una construcción específica del pasado histórico?

Si la reconstrucción del pasado histórico que se presenta en los textos es memoria episódica, comprometida emocionalmente con una postura concreta, estaremos en presencia de diversas construcciones desde y para diversas ideologías. Estas creencias son acerca de una cosa, creemos que algo es verdadero, atractivo o detestable (Van Dijk 2005:38) y, en función de esos procesos, generamos discursos, en este caso discursos académicos, discursos históricos. La memoria compone una historia, Van Dijk no la considera una creencia, pero en este caso las genera al ser legitimadas académicamente a través del discurso del texto y del discurso del docente.

Van Dijk (2006) establece que estas proposiciones discursivas generan redes, definidas como una colección de nodos relacionados por senderos 0 , más específicamente, como gráficos con bordes ( $p .40)$. En el caso que me ocupa, Franceschi y Domínguez hablan de "intentonas militares". El nodo "intentona" está conectado con el nodo "militares" y con el verbo enfrentar que de alguna forma muestran una red de proposiciones de contenido. La intentona no tiene autor ni responsable, por lo tanto esta red puede relacionarse con muchas otras que no necesariamente describen qué ocurrió, cómo, ni por qué.

Lo descrito genera una red que, según Van Dijk (2006), es equivalente a una lista o esquema organizado de preposiciones, que refuerza las conexiones entre cada nodo, en este caso una visión "gris" y poco profunda de un golpe de estado que evidenció una situación del país y que colocó en la mira pública al actual presidente de Venezuela.

En el segundo texto citado, Gómez (2007) describe previamente la situación social vivida en el país, que sin duda prepara o justifica los estallidos sociales. En este sentido, las creencias del autor constituyen un "mundo según nosotros" (Van Dijk, 2006), es decir, los nodos no son solamente presentados como en el caso anterior, sino que el autor hace una proyección socialmente controlada de sus creencias, al en alguna medida "justificar" o "explicar" el golpe de estado, que en este caso identifica no sólo a su cabecilla sino a los demás participantes.

En todo caso, se trata de una manipulación en ambos casos, en uno por omisión y en el otro por justificación, redundando ambos en el ámbito académico en la adquisición, construcción y modificación de creencias sociales. La ideología de los autores genera creencias en los estudiantes, y éstas generan a su vez ideología.

Puede de igual forma tratarse de memorias episódicas, definidas por Van Dijk (2006) como aquella parte de la memoria en la que se 
almacenan las creencias sobre episodios concretos (hechos, eventos, situaciones, etc.) de los que se ha sido testigo o en los que se ha participado. Los hechos descritos por los autores forman parte de la historia contemporánea reciente que hoy reclama y castiga posturas concretas, de tal forma que los registros en los textos del golpe de estado protagonizado por el actual presidente pueden haberse realizado desde la memoria episódica o personal que no sólo se concreta a la participación física en los hechos sino al compromiso ideológico con los mismos.

El tercer y último texto citado, de igual forma describe de manera clara la situación social, económica y política del país para luego hablar del golpe de estado. Se describe el hecho específico: tiempo, participantes y acciones, partiendo de las creencias particulares del autor, pero que desde la palestra del texto y el discurso del docente se vuelve creencia general. Así como en los casos citados por Van Dijk (2006), el holocausto, la guerra civil en Bosnia, entre otros, el conocimiento personal en la memoria episódica del autor representa experiencias personales, los hechos descritos pueden ser prototipo de experiencias grupales y ser representados en la memoria social. Este ejemplo, a través del cual he descrito el abordaje de los hechos históricos en los libros de textos mencionados, es uno de muchos que en el cuerpo de la tesis doctoral son descritos en profundidad y que tienen como denominador común la presencia de elementos ideológicos parcializados a favor o en contra en diferentes contextos y que demuestran la necesidad de estas reflexiones.

Finalmente, y luego de haber realizado esta aproximación a las categorías: conocimiento, creencia, emoción, desde los aportes de Van Dijk, puedo generar las siguientes apreciaciones:

- En Historia, como en otras áreas del saber, no existe lugar para la ingenuidad en la escogencia de sustantivos, adjetivos, nodos temáticos. La estructuración de un determinado discurso académico descansa sobre una red de complejos elementos que de forma ineludible se tiñe de la ideología de quien la construye.

- Los libros de texto de historia contemporánea de Venezuela responden a un discurso de poder. Un discurso de élite del autor y la editorial que legitiman y permiten dichos discursos.

- El docente dentro del aula hace uso de esas redes discursivas y también añade las propias.

- El alumno recibe estos discursos eminentemente ideológicos que van configurando conocimiento, memoria social, creencias e ideología.

Finalmente cabe preguntarse si el papel que está llamada a cumplir la educación, la enseñanza de la Historia, ¿es la réplica de posturas ideológicas "casadas" con la legitimación y deslegitimación de posturas y visiones de la realidad? ¿Cómo construir un discurso multívoco que 
permita que todas las memorias episódicas estén representadas sin marginar a ninguna? ¿Cómo prescindir de la ideología cuando es inevitable hablar y escribir desde una? ¿Cuál es la correcta? ¿Dónde está el verdadero conocimiento histórico?

Las respuestas a estas y otras interrogantes son el objeto de mi tesis doctoral en la que espero generar un aporte teórico que oriente la enseñanza de la Historia hacia la formación de alumnos y docentes críticos, tolerantes, abiertos y justos.

\section{Referencias bibliográficas:}

- Arias Amaro, A. (2000). Lecciones de historia contemporánea de Venezuela. Editorial COBO. Caracas, Venezuela.

- Franceschi, N. y Domínguez, F. (2005). Historia contemporánea de Venezuela. Editorial Colegial Bolivariana. Caracas, Venezuela.

- Gómez, A. (2007). Historia contemporánea de la República Bolivariana de Venezuela. Editorial Santillana. Caracas, Venezuela.

- Van Dijk, T. (2006). Ideología: una aproximación multidisciplinaria. Editorial Gedisa. Barcelona, España. 